\title{
Pulse-actuated micromechanical resonator using digital Kalman observer
}

\author{
Eric Colinet, Dimitri Galayko and Jérôme Juillard \\ Supélec, Gif-sur-Yvette, France \\ dimitri.galayko@supelec.fr
}

\begin{abstract}
The paper presents a digital oscillating loop for a MEMS resonator in which the instantaneous resonator position is estimated on-line with a Kalman observer. The approach is applied to an oscillating loop with a pulse-actuated umechanical resonator, where the predicted resonator position can be used for oscillation amplitude control or resonator parameters measurement. We demonstrate the very good immunity of the method toward internal system and measurement noises.
\end{abstract}

Keywords: Resonant sensor, Kalman observer, estimation, sigma-delta conversion

\section{Introduction}

Resonant micro-electro-mechanical sensors are wellsuited for measurement and detection applications, since their natural resonance frequency is highly sensitive to exogenous parameters (temperature, pressure, acceleration...). The resonance frequency is detected while the structure is brought to auto-oscillations thanks to a positive feedback scheme.

Different approaches make it possible to bring a resonator to oscillations. The short-pulse actuation [1] of resonant MEMS ideally reduces the sensitivity toward actuator nonlinearities and capacitive crosstalk. The governing idea is to excite the system with a brief pulsed force whenever the resonator passes through the equilibrium position (fig. 1): thus, the amplitude of the actuating force is independent of the position of the resonator; this makes this method particularly suitable for electrostatically-actuated resonators and, more generally, for resonators with displacement-dependent nonlinearities. Another advantage of the method is that the only critical analog component it requires is an inexpensive one-bit ADC (comparator). Finally, the actuating force takes only two non-zero values corresponding to positive and negative pulses, so that the method is insensitive to the voltage nonlinearity of electrostatic actuation.

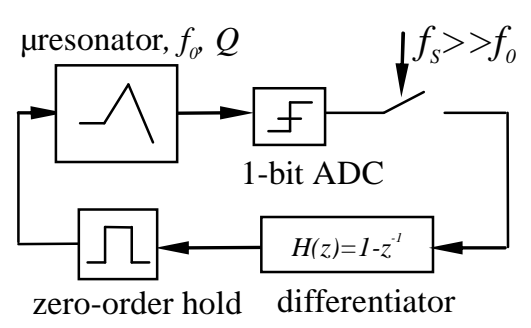

Figure 1. Short-pulse actuated resonator in oscillating loop.

However, if a measurement noise disturbs the signal at the input of the comparator, the equilibrium position cannot be detected properly. In this case the resonator can be excited at a non-zero position and the linearity and sensitivity of the sensor decrease. A natural solution to this problem would be to filter out measurement noise (fig. 2). However, a conventional filter would introduce a delay so that the pulses would not be generated at zero resonator position. Another drawback of such solution is that it requires high-resolution ADCs, which can be costly to integrate in a MEMS context.

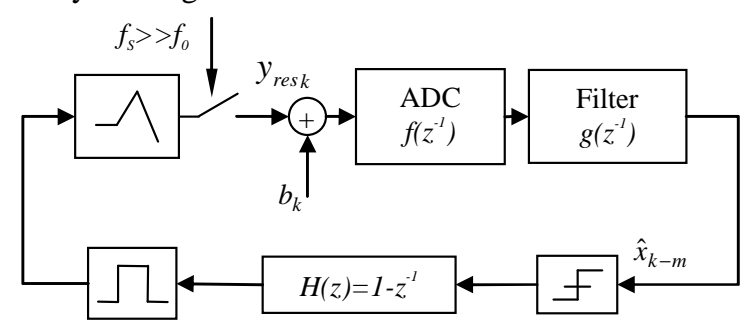

Figure 2. Straightforward approach of oscillation control. The ADC and the filter introduce an $m$ clock cycles delay.

In the present paper, we propose an architecture for the short-pulse actuation of micro-resonators using digital Kalman observer. A Kalman observer, also called Kalman filter, estimates the current resonator position from the noisy ADC input, whatever the measurement delay is. To be efficient such a solution requires also a high-resolution ADC: however, we propose an implementation of our architecture in which a $\Sigma \Delta$ ADC is used - the only critical analog component in a $\Sigma \Delta$ 
ADC being a one-bit comparator [2-3]. Even though such an ADC introduces some delay in the feedback loop, this is accounted for by the Kalman observer.

Real-time knowledge about the resonator state offers many possibilities for measurement and control on the sensor, for example, an oscillation magnitude control or measurement of resonator parameter variation (spring constant, damping...).

\section{Kalman estimation of the resonator position}

A "discrete-time Kalman observer" realizes a statistical estimation of the internal states of a noisy linear system [4-5]. Let's the system be described by its state equations:

$\left\{\begin{array}{c}x_{k}=A x_{k-1}+B u_{k-1} \\ y_{k}=C x_{k}+D u_{k}\end{array}\right.$

where $x_{k}$ denotes the system internal state at $k$-instant, $u_{k}$ is the input signal, $y_{k}$ is the system output signal, $A, B$, $C, D$ are constant matrixes. The first equation is the system state equation describing the system dynamics. The second is the measurement equation defining the relation between the measurable output and the system internal state.

A noise can disturb the state equation and the measurement equation:

$\left\{\begin{array}{c}x_{k}=A x_{k-1}+B u_{k-1}+f_{k-1} \\ y_{k}=C x_{k}+D u_{k}+g_{k}\end{array}\right.$,

where $f_{k}$ and $g_{k}$ are zero-centered noise vectors with variances $F$ and $G$ and joint covariance $M$. Observation of such a system (i. e. measurement of $y$ ) does not give a complete information about the system state because of the noise components.

A Kalman observer makes it possible to estimate $x_{k}$ or to predict a future state $x_{k+m}$ with minimal estimation error. The observer uses the following information:

- $\quad$ Precise system model (matrices $A, B, C, D$ );

- $\quad$ The system input $u_{k}$, and $u_{k-1}$,

- The system output $y_{k}$.

The Kalman estimation includes two steps. Assume the state $\hat{x}_{k-1}$ at instant $k-1$ is somehow known, with an error variance $P_{k-1}$. An a priori value of the state at instant $k\left(\hat{x}_{k}^{-}\right)$can be obtained:

$\hat{x}_{k}^{-}=A \hat{x}_{k-1}+B u_{k-1}$

The measured value $y_{k}$ is then used to update the state at instant $k$. The additive correction of the a priori estimated state at $k$ is proportional to the difference between the a priori output at instant $k$ defined as $\hat{y}_{k}^{-}=C \hat{x}_{k}^{-}+D u_{k}$ and the measured $y_{k}$ :

$\hat{x}_{k}=\hat{x}_{k}^{-}+K_{k} \cdot\left(y_{k}-\hat{y}_{k}^{-}\right)$,

where $K_{k}$ is the Kalman gain which guarantees the minimal variance of the error $x_{k}-\hat{x}_{k}$, given all available information about the system and statistical properties of the disturbing noises (matrixes $F, G$ et $M$ ). The calculation of the Kalman gain is described in detail in the literature [4], [5].

In this way, a Kalman observer is a dynamical system defined by the state equation

$\hat{x}_{k}=A \hat{x}_{k-1}+B u_{k-1}+K_{k} \cdot\left(y_{k}-C\left(A \hat{x}_{k-1}+B u_{k-1}\right)\right)$

Or, putting it into a canonical form (1):

$\hat{x}_{k}=\left(A-K_{k} C B\right) \hat{x}_{k-1}+\left[\begin{array}{lll}B & -K_{k} C B & K_{k}\end{array}\right]\left[\begin{array}{c}u_{k-1} \\ u_{k} \\ y_{k}\end{array}\right]$

Also, at each step the variance of the error on $\hat{x}_{k}\left(P_{k}\right)$ is calculated; it is used for calculation of the Kalman gain at the next step.

Since the state variables describe completely the system state at instant $k$, from the estimated $\hat{x}_{k}$ it is possible to find any other depending parameter $\theta$ related to the state variable with a known function $f\left(\hat{x}_{k}\right)$.

\section{Resonator model}

A resonant sensor can be modeled as a second-order continuous-time single-input single-output system which can be characterized by a Laplace domain transmission characteristic :

$$
y_{\text {res }} / u=H(s)=\frac{\omega_{0}}{s^{2}+s \omega_{0} / Q+\omega_{0}^{2}},
$$

where $u$ is the input force, $\mathrm{y}_{\text {res }}$ is the resonator position, $\omega_{0}$ is the natural resonance frequency, $Q$ is the quality factor.

However, given that the sensor is driven by digital pulses, we model it as discrete-time system

$y_{\text {res }} / u=H\left(z^{-1}\right)=\frac{b_{2} z^{-2}+b_{1} z^{-1}}{a_{2} z^{-2}+a_{1} z^{-1}+1}$.

The correspondence between $\omega_{0}, Q$ et $a_{1}, a_{2}, b_{1}, b_{2}$ is defined with zero-order hold continuous time to sampled 
time transformation.

In state-variable representation (1), the resonator is characterized by the following matrices:

$A_{\text {res }}=\left[\begin{array}{cc}1 & 0 \\ a_{1} & a_{2}\end{array}\right], B_{\text {res }}=\left[\begin{array}{lll}0 & 1\end{array}\right], C_{\text {res }}=\left[b_{2} b_{1}\right], D_{\text {res }}=[0]$.

\section{Sigma-delta modulator}

A sigma-delta modulator achieves a low-bit analogto-digital conversion preserving a high resolution only in a limited band [6]. A one-bit second-order lowpass sigma-delta modulator architecture is shown in Figure 3a. It includes two integrators and a 1 bit ADC (comparator). The modulator can be modelled by two linear filtering transfer functions: second-order highpass for the quantization noise (noise transfer function, NTF) and second-order low-pass for the signal (signal transfer function, STF) (Figure 3b). So, at low frequencies, the quantization noise is reduced whereas the signal passes through. To recover the original signal, the noise must be eliminated by a digital filtering.

The integrators used in our model had the following transfer functions:

$H_{1}\left(z^{-1}\right)=\frac{0.5 z^{-1}}{1-z^{-1}}, H_{2}\left(z^{-1}\right)=\frac{z^{-1}}{1-z^{-1}}$.

The NTF and STF are expressed as:

$$
\begin{aligned}
& \operatorname{STF}\left(z^{-1}\right)=\frac{0.5 z^{-1}}{1-z^{-1}+0.5 z^{-2}}, \\
& \operatorname{NTF}\left(z^{-1}\right)=\frac{1-2 z^{-1}+z^{-2}}{1-z^{-1}+0.5 z^{-2}} .
\end{aligned}
$$

\section{Global system presentation}

The global system is presented in Figure 4. As one can see, after the sigma-delta modulation, the signal is noisy and $z^{-2}$ delayed. The relation between the output of the modulator and the resonator input force can be written as:

$$
y=\left(H\left(z^{-1}\right) u+b\right) \cdot \operatorname{STF}\left(z^{-1}\right)+w \cdot \operatorname{NTF}\left(z^{-1}\right) .
$$

The resonator output $y_{\text {res }}=H\left(z^{-1}\right) u$ is reconstructed from $y$ with a Kalman observer. To achieve it, a state variable model of the overall system is built: the three secondorder blocs of the system yield a sixth-order system equations in the form (1). The Kalman observer built as explained in section II is a sixth order system in which the first two components of the state vector $X$ represent the estimated resonator state, the four others represent the internal state of the sigma-delta blocs (NTF and STF). Since we need to know the resonator position at instant $k$, the output equation of the Kaman observer is the following:

$$
\hat{y}_{\text {resk }}=\left[\begin{array}{lllll}
C_{r e s} & 0 & 0 & 0 & 0
\end{array}\right] \cdot X \text {. }
$$

This estimated resonator position is then used to generate short input pulses as in the system of fig. 1 .

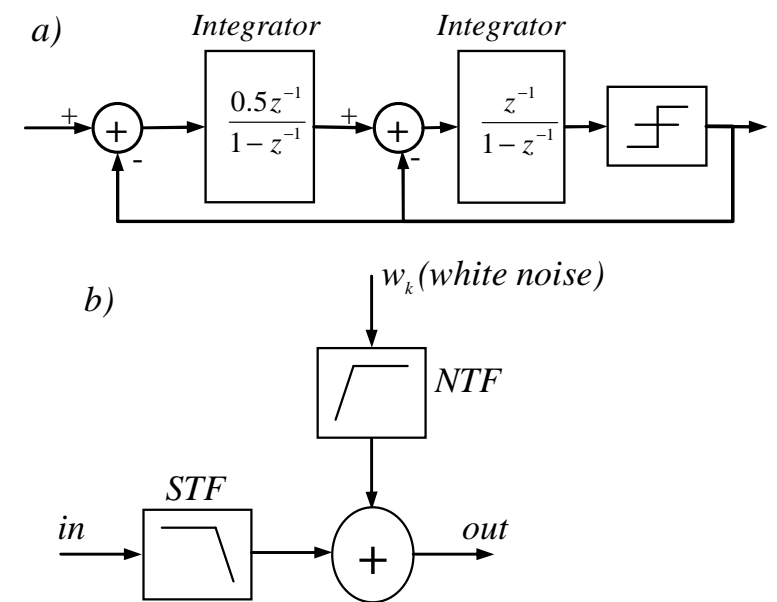

Figure 3. Sigma-delta modulator: a) architecture, b) linearized model.

\section{Simulation results}

The natural frequency $f_{0}$ of the resonator was set to 0.05 (normalized with respect to the sampling frequency) and the quality factor $Q$ to 100 . This yields an over-sampling ratio of 20 resulting in rather poor resolution of the $\Delta \Sigma \mathrm{C}$. To test the noise immunity of the designed system, a white-noise was added at the resonator output. Its standard deviation was of the same order of magnitude as the power of the resonator output signal.

The obtained results proved two fundamental benefits from the use of Kalman observer.

Resonator position estimation. Matlab simulations show that, in the absence of resonator state noise $f_{k}$ (eq. 2 ), the reconstructed resonator position is almost identical to the real one (Figure 5), the value of the estimation error is about $10^{-10}$, i. e. comparable with the simulation tolerance. Thus the generated pulses happen exactly at the equilibrium position instants, in the same way as in the noiseless system of fig. 1. So, thanks to the Kalman observer, the AD converter becomes a completely transparent block.

Additive output noise filtering. In the system of fig. 1 , the additive noise $b_{k}$ would yield parasitic switching and the system would never be brought to oscillate. On the other hand, our architecture shows excellent 
immunity to high levels of measurement noise, and is similar in performance to techniques based on PLLs, while preserving the advantages of short-pulse actuation.

\section{Kalman estimation of sensor state : practical aspects}

Important condition for successful operating of a Kalman observer is a very precise knowledge about the observed system. This explains the "perfect" aspect of our results: since no state noise disturbed the resonator $(F=0)$, we had the complete information about the system. Moreover, since the input was also known, the Kalman observer could perfectly model the system behaviour.

If a state noise exists, the residual error will be a white noise with non-zero variance $P$. In the applications using short-pulse actuation this doesn't happen, since the resonator is submitted only to known forces generated by the input transducer.

However, for example, in accelerometer or gyroscope applications, the input force can be considered to have random disturbing components which add to the input transducer force. They can be considered as a state noise, thus a Kalman observer can be used to estimate the resulting resonator state with a minimal error.

A Kalman estimation is problematic when the resonator model is defined with tolerances. If the measurement noise is very high, which is the case with a sigma-delta modulator, the state estimation will be achieved with a residual non-white error strongly correlated with the real resonator state position. This situation is demonstrated in the plot of Figure 5, where the resonator model used for the construction of the Kalman observer had a 5\% error on the natural resonance frequency. A precise position estimation is not possible, thus the pulses are generated at non-zero position making impossible to realize an accurate resonator excitation.

This shows the importance of the a priori knowledge about the resonator model. Different methods exist to identify on-line a resonant sensor [7]. One possible solution is to use a non-linear adaptative Kalman observer: the unknown state variables are the system coefficients together with the original state variables [5]. This solution is the subject of the ongoing work.

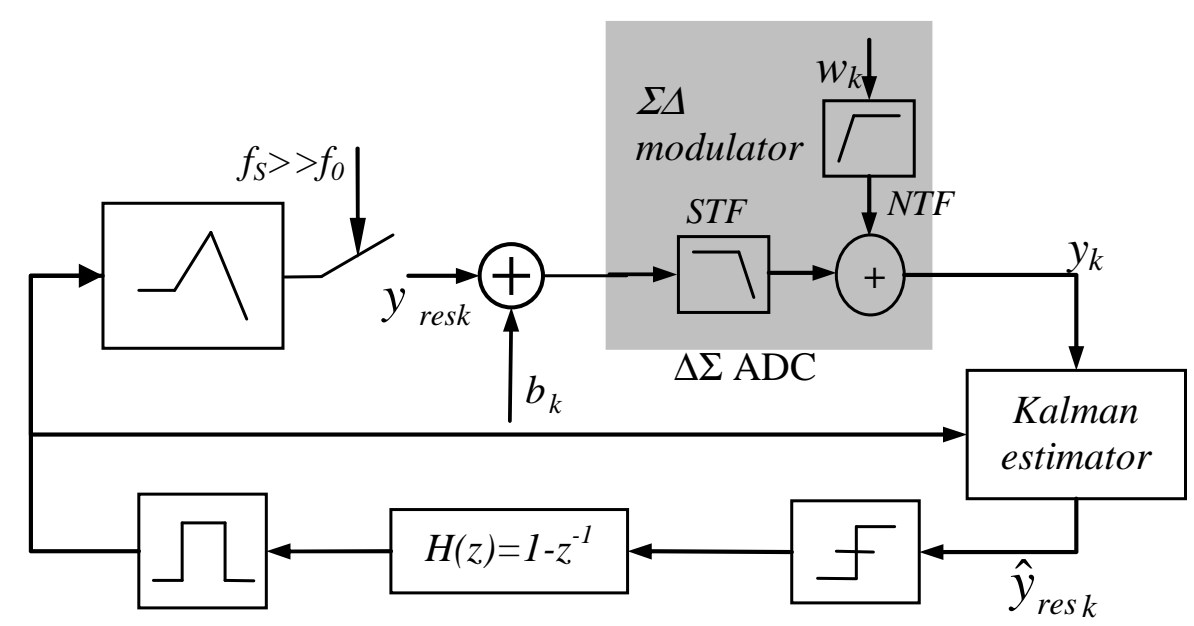

Figure 4. Proposed architecture using Kalman observer and sigma-delta conversion. At instant $k$, the ADC output is equal to its input at instant $k$-2 plus some conversion noise $w_{k}$. However, the Kalman observer calculates the estimate $\hat{y}_{\text {res } k}$ of the resonator position $y_{\text {res } k}$. Provided the global system is accurately modeled, the reconstruction error can be minimized to very weak values predicted by the optimal filtering theory. 

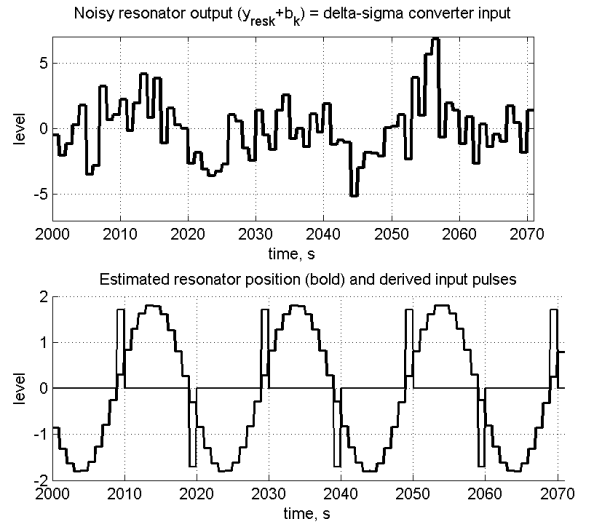

Delta-sigma outpu
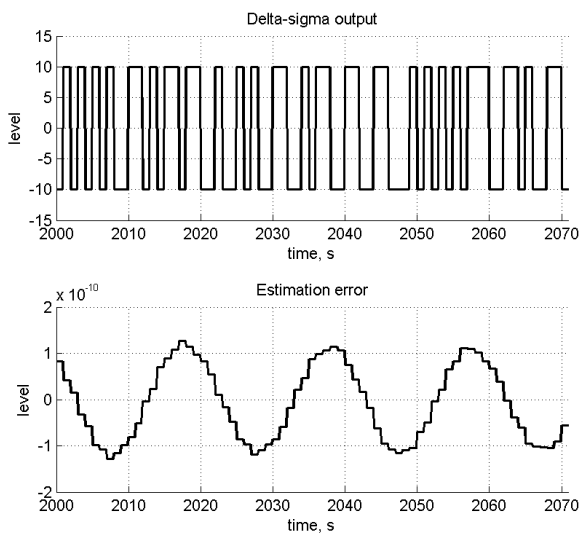

Figure 5. Simulation results: the resonator model is precisely known. Very low estimation error level.

\section{Conclusions and perspectives}

The results show the very high potential of the on-line estimation of resonant sensor state. This information can be used, for example, for oscillation amplitude control. However, the study pointed out the high sensibility of the method toward the error on the resonator model. It is evident, that the presented system should be initialized with the correct values of the resonator parameters. If the resonator parameters change during the operating, an on-line identification method, such as adaptative Kalman filtering, should be used. The study of the latter approach is the subject of the ongoing work.
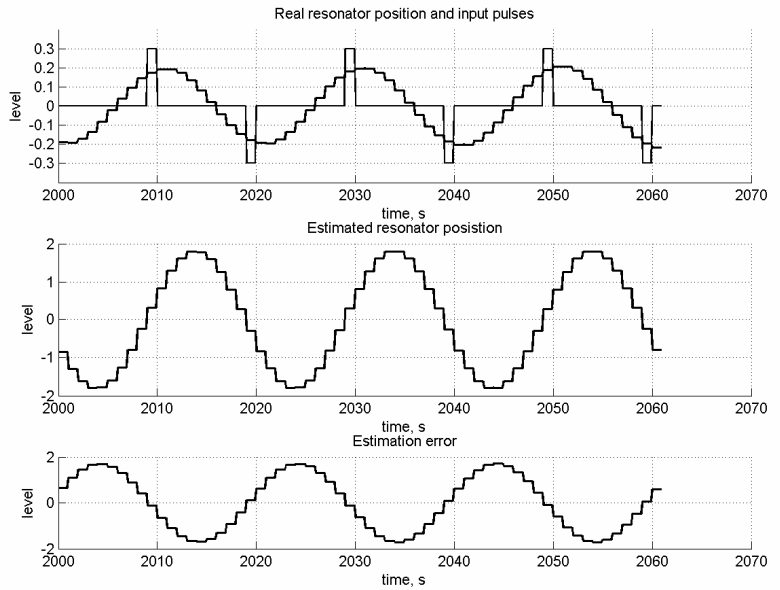

Figure 6. Simulation results. 5\% of model error on the resonator natural frequency: a substantial estimation error is observed.

\section{References}

[1] Colinet et al, "Actuation of resonant MEMS using short pulsed forces", Sensors \& Actuators A 155 (2004), 118-125.

[2] Colinet et al., "Resolution enhancement of a sigma-delta microaccelerometer using signal prediction" International Conference on MEMS, NANO and Smart Systems, 409-413, 2004.

[3] Kraft. et al. "Closed loop micromachined inertial sensors with higher order sigma-delta modulators". In proceedings of the International Conference on modeling and Simulation of Microsystems, 104-107, 2001.

[4] Franklin et al., "Digital Control of Dynamic Systems", Second Edition, Addison-Wesley, 1990.

[5] Robert F. Stengel, Optimal control and estimation, Dover Publications, INC, New York 19

[6] S. Norsworthy et al, "Delta-Sigma Data Converters. Theory, Desing, and Simulation”. IEEE Press, Piscataway 1997

[7] Torsten Söderström, Petre Stoica, System identification, Prentice hall, 1989 